AUTOR

Luiz Gustavo Oliveira Brito

ORIENTADOR

Prof. Dr. Heitor Ricardo Cosiski

MARANA

Resumo de tese

Palavras-chave

Câncer de mama

HIF-1

Quimioterapia neoadjuvante

Status axilar

Fatores prognósticos

Keywords:

Breast cance

HIF-1

Neoadjuvant chemotherapy

Axillary status

Prognostic factors

Expressão imunohistoquímica do fator indutor de hipóxia 1-alfa (HIF-1 $\alpha)$ em pacientes com câncer de mama localmente avançado

Immunobistochemical expression of bypoxia-inducible factor 1-alpha (HIF-1 $\alpha)$ in locally advanced breast cancer patients.

Dissertação de Mestrado apresentada ao Programa de Pós-Graduação em Ginecologia e Obstetrícia da Faculdade de Medicina de Ribeirão Preto da Universidade de São Paulo, para obtenção do Título de Mestre, em 15 de julho de 2010.

OBJETIVOS: determinar a expressão imunohistoquímica do fator indutor de hipóxia 1 -alfa $(H I F-1)$ e suas variáveis associadas em pacientes com câncer de mama localmente avançado.

MÉTODOS: vinte e sete mulheres foram biopsiadas para diagnóstico histopatológico do carcinoma mamário e submetidas a tratamento quimioterápico pré-cirúrgico. Analisou-se a associação do HIF-1 com idade, tamanho tumoral, grau histológico, estadio clínico, status hormonal e axilar, resposta clínica e patológica após tratamento quimioterápico, expressão do receptor de estrogênio, progesterona e cerbB2.

RESULTADOS: a expressão de HIF-1 foi presente em $66,7 \%$ das pacientes. $\bigcirc$ único fator associado à sua presença foi o status axilar positivo $(p=0,02)$, tendo permanecido durante a análise univariada (RR bruto=1,9). As demais variáveis não apresentaram associação estatisticamente significante.

CONCLUSÃO: existe uma associação estatisticamente significante entre o acometimento linfonodal e a presença de HIF-1 em pacientes com câncer de mama localmente avançado.

AUTOR

Paulo César Praciano de Sousa

ORIENTADOR

Prof. Dr. Francisco EdSON dE

LuCENA Feitosa

\title{
Avaliação da perda sanguínea em gestantes submetidas à indução do parto com misoprostol
}

Evaluation the blood loss in pregnancies with misoprostol induced labor

Resumo de tese

Palavras-chaves:

Trabalho de parto induzido

Misoprosto

Hemorragia pós-parto

Índices de eritrócitos

Parto vaginal

Keywords:

Induced labour

Misoprostol

Postpartum blood

Erythrocyte indices

Vaginal delivery.
Dissertação de Mestrado apresentada ao Programa de Pós-Graduação em Tocoginecologia da Universidade Federal do Ceará, em 3 de setembro de 2009.

OBJETIVOS: avaliar a perda sanguínea em partos vaginais induzidos pelo misoprostol e em cesáreas com tentativa prévia de indução do parto pelo misoprostol. MÉTODOS: este estudo foi aprovado pelo Comitê de Ética em Pesquisa da Maternidade-Escola Assis Chateaubriand - UFC. As pacientes assinaram um termo de consentimento informado. Realizouse estudo em 101 gestantes com indicação para indução do trabalho de parto, que foram avaliadas pela dosagem de hemoglobina pré e pós-parto para estimativa da perda sanguínea no parto. Procedeu-se à indução do trabalho de parto com misoprostol $25 \mathrm{mcg}$, via vaginal, a cada 6 horas, em um número máximo de seis. $\bigcirc$ grupo controle foi composto por 30 pacientes que entraram em trabalho de parto espontaneamente e por 30 pacientes que se submeteram à cesárea eletivamente. $\bigcirc$ estudo da hemoglobina foi avaliado através de ANOVA para medidas repetidas, onde foram verificados o efeito do tempo (pré e pós-parto) e o efeito do grupo (com e sem uso do misoprostol). RESULTADOS: Existem diferenças significativas entre os níveis hemoglobina pré e pós-parto $(\mathrm{p}<0.0001)$ nos partos vaginais induzidos pelo misoprostol $(1,6+1,4 \mathrm{mg} / \mathrm{dl})$, nos partos vaginais não induzidos $(1,4+1,0 \mathrm{mg} / \mathrm{dl})$, nas ceráreas com tentativa prévia de indução $(1,5+1,0 \mathrm{mg} / \mathrm{dl})$ e nas cesáreas eletivas $(1,8+1,1 \mathrm{mg} / \mathrm{dl})$. Porém as diferenças são proporcionais em ambos os grupos, ou seja, a diferença ocorre tanto no grupo que fez uso do misoprostol quanto no grupo que não fez uso do misoprostol, tanto na cesárea $(p=0.6)$ quanto no parto normal ( $p=0.2)$. CONCLUSÃO: a indução do parto com misoprostol não altera a perda sanguínea durante o parto, tanto nos partos vaginais induzidos, quanto nas cesáreas com tentativa prévia de indução. 